\title{
Analytical solution of linearized equations of the Morris-Lecar neuron model at large constant stimulation
}

\author{
A.V. Paraskevov ${ }^{1,2}$, T.S. Zemskova ${ }^{3,4}$ \\ ${ }^{1}$ National Research Centre "Kurchatov Institute", 123182 Moscow, Russia \\ ${ }^{2}$ Institute for Information Transmission Problems of the Russian Academy of Sciences, 127051 Moscow, Russia \\ ${ }^{3}$ Ecole Polytechnique, 91128 Palaiseau, France \\ ${ }^{4}$ Moscow Institute of Physics and Technology (National Research University), 141700 Dolgoprudny, Russia
}

\begin{abstract}
The classical biophysical Morris-Lecar model of neuronal excitability predicts that upon stimulation of the neuron with a sufficiently large constant depolarizing current there exists a finite interval of the current values where periodic spike generation occurs. Above the upper boundary of this interval there is a four-stage damping of the spike amplitude: 1) minor primary damping, which reflects a typical transient to stationary state, 2) plateau of nearly undamped periodic oscillations, 3 ) strong damping, and 4) reaching a constant stationary asymptotic value $V_{s t}$ of the neuron potential. We have linearized the Morris-Lecar model equations at the vicinity of $V_{s t}$ and have shown that the linearized equations can be reduced to a standard equation for exponentially damped harmonic oscillations. Importantly, all coefficients of this equation can be explicitly expressed through parameters of the original Morris-Lecar model, enabling direct comparison (i.e. without any fitting) of the numerical and analytical solutions for the neuron potential dynamics at later stages of the spike amplitude damping. This allows to explore quantitatively the applicability boundary of linear stability analysis that implies exponential damping.
\end{abstract}

Keywords: neuronal dynamics, Morris-Lecar model, constant current stimulation, periodic spiking, damped oscillations 


\section{Introduction}

The Morris-Lecar model [1,2] is a classical biophysical model of spike generation by the neuron, which takes into account the dynamics of voltage-dependent ion channels and realistically describes the spike waveform. The model predicts that upon stimulation of the neuron with a sufficiently large constant depolarizing current $I_{\text {stim }}$, there exists a finite interval of $I_{s t i m}$ values where periodic spike generation occurs [2-5]. Numerical simulations have shown that in the Morris-Lecar model the cessation of periodic generation of spikes above the upper boundary of this interval (i.e. at $I_{\text {stim }}>I_{\max }$ in Fig. 1) occurs through a damping of the spike amplitude, arising with a delay inversely proportional to the value of $I_{\text {stim }}[6]$. In particular, the damped dynamics can be divided into four successive stages: 1) minor primary damping, which reflects a typical transient to stationary dynamic state, 2) plateau of nearly undamped periodic oscillations, which determines the aforementioned delay, 3) strong damping, and 4) reaching a constant stationary asymptotic value $V_{s t}$ of the neuron potential. As the last two stages resemble the well-known exponentially-damped harmonic oscillations, we tackled to find an analytical description for these.

In this paper, we have linearized the Morris-Lecar model equations at the vicinity of the asymptote $V_{s t}$. The resulting equations have been then reduced to an inhomogeneous Volterra integral equation of the second kind. In turn, the latter has been transformed into an ordinary differential equation of the second order with a time-dependent coefficient at the first-order derivative. As this time dependence was just an exponential decay with the small preexponential factor, we considered its asymptotic value and analytically solved the final equation. In order to verify the analytical solution found, we have compared it with the numerical solution obtained using the standard MATLAB tools for systems of ordinary differential equations (see Supplementary Material, which contains the MATLAB codes and generated data used for the Figures).

As the result, we have accurately shown that the linearized system of equations of the Morris-Lecar model can be reduced to a standard equation of exponentially damped harmonic oscillations for the neuron potential. Since all coefficients of this equation are explicitly expressed through parameters of the original Morris-Lecar model, one can directly (i.e. without any fitting) compare the numerical and analytical solutions for dynamics of the neuron potential at last two stages of the spike amplitude damping (left graphs in Fig. 2 and Fig. 3). The results allow a quantitative study of the applicability boundary of linear stability analysis that implies exponential damping.

\section{Standard Morris-Lecar model}

As phase plane analysis of the Morris-Lecar (ML) model is extensively described in the most of textbooks on mathematical neuroscience (e.g., [7-11]), we omit it and provide only basic facts on the model, and its formal description.

Qualitatively, the classical two-dimensional ML model [1,2] (cf. [12]) couples dynamics of the transmembrane potential $V$ of the neuron with dynamics of the transmembrane conductance $w$ of potassium ions. Spikes represent characteristic pulses of $V$ (see the gray inset in Fig. 1, in the range from $I_{\min }$ to $I_{\max }$ ). In the ML model the rate of change of $V$ depends on the current value of $w$ in such a way that dynamics of $w$ provides a negative feedback with respect to the dynamics of $V$. In turn, the rate of change of $w$ is proportional to the difference between the current value of $w$ and some "asymptotic" value $w_{\infty}$, which nonlinearly depends on $V$. As a result, $w$ tends to reach $w_{\infty}$, which is changed itself in time due to dynamics of $V$. If one neglects the relaxation of $w$ to $w_{\infty}$, i.e. assumes that it occurs instantly, then the generation of spikes in the ML model does not occur. The upper value of the stimulating current, $I_{\max }$, above which the continuous periodic generation of spikes stops, corresponds to the onset of a fast (compared to the rate of change of $V$ ) relaxation of $w$ to $w_{\infty}$.

Quantitatively, standard equations of the ML model for dynamics of the neuronal potential $V$ and for relaxation dynamics of the normalized conductance $w$ of potassium ions are given by

$$
\left\{\begin{array}{l}
C_{m} d V / d t=-I_{i o n}(V, w)+I_{s t i m}, \\
d w / d t=\left(w_{\infty}(V)-w\right) / \tau(V),
\end{array}\right.
$$

where the total sum of ion currents

$$
I_{i o n}(V, w)=g_{C a} \cdot m_{\infty}(V) \cdot\left(V-V_{C a}\right)+g_{K} \cdot w \cdot\left(V-V_{K}\right)+g_{L} \cdot\left(V-V_{L}\right),
$$

$I_{\text {stim }}$ is an external stimulating current, and the constituent functions

$$
\begin{aligned}
m_{\infty}(V) & =\frac{1}{2}\left[1+\tanh \left(\left(V-V_{1}\right) / V_{2}\right)\right], \\
w_{\infty}(V) & =\frac{1}{2}\left[1+\tanh \left(\left(V-V_{3}\right) / V_{4}\right)\right], \\
\tau(V) & =\tau_{\max } / \cosh \left(\left(V-V_{3}\right) /\left(2 V_{4}\right)\right) .
\end{aligned}
$$




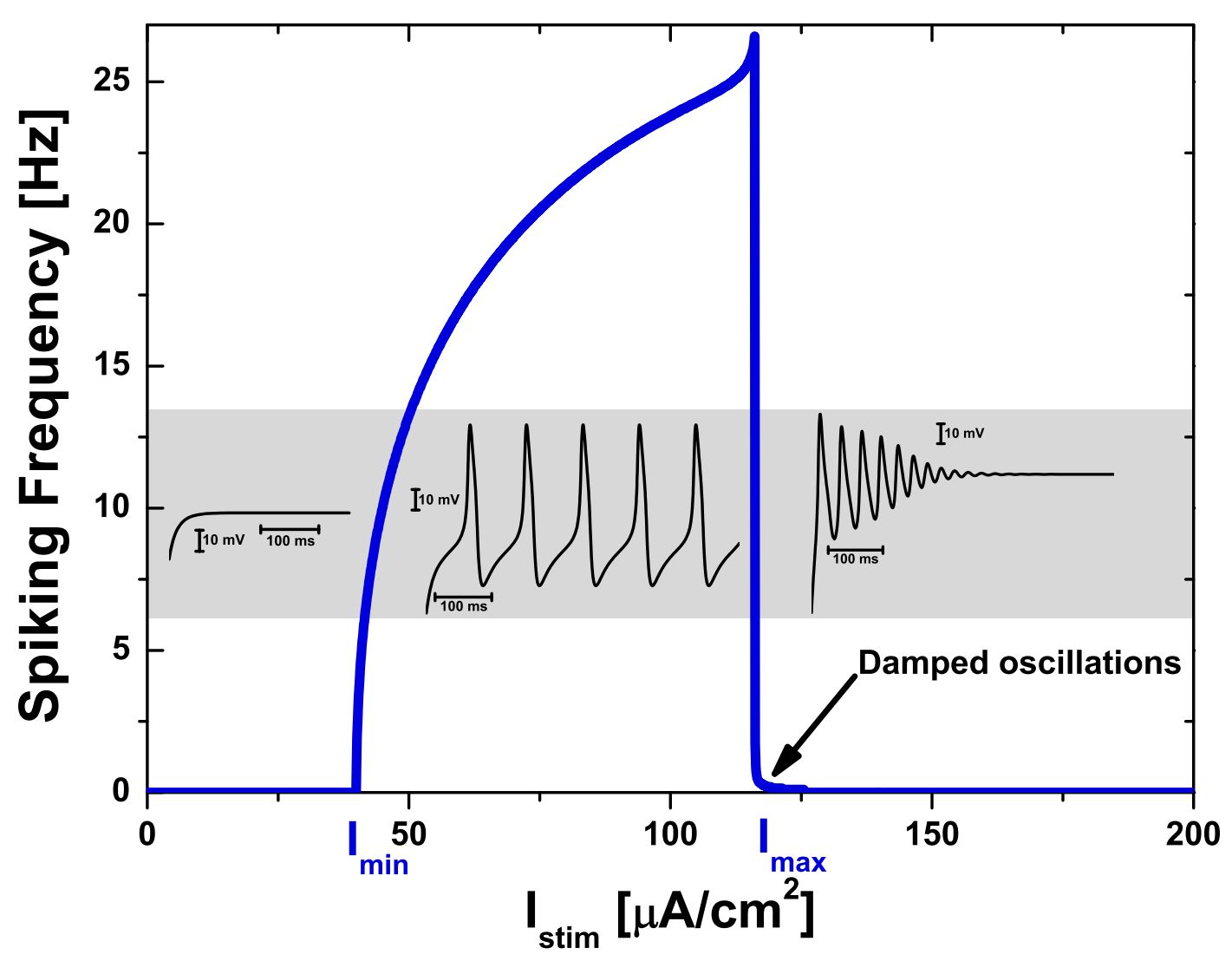

Figure 1. Dependence of spike generation frequency (averaged over fixed time interval of $20000 \mathrm{~ms}$ ) on constant stimulating current $I_{\text {stim }}$ and, on the gray inset, typical examples of dynamics of the neuron potential in the corresponding ranges of $I_{\text {stim }}$ values for the Morris-Lecar model with the 1st excitability type [2]. Spikes are characteristic pulses of the neuron potential (see the gray inset in the range from $I_{\min }=40 \mu \mathrm{A} / \mathrm{cm}^{2}$ to $I_{\max }=116.1 \mu \mathrm{A} / \mathrm{cm}^{2}$ ).

For numerical simulations we have used the following values of the ML model parameters corresponding the 1st neuronal excitability type [2]: $C_{m}=20 \mu \mathrm{F} / \mathrm{cm}^{2}, g_{C a}=4 \mathrm{mS} / \mathrm{cm}^{2}, g_{K}=8 \mathrm{mS} / \mathrm{cm}^{2}, g_{L}=2 \mathrm{mS} / \mathrm{cm}^{2}, V_{C a}=120 \mathrm{mV}$, $V_{K}=-84 \mathrm{mV}, V_{L}=-60 \mathrm{mV}, V_{1}=-1.2 \mathrm{mV}, V_{2}=18 \mathrm{mV}, V_{3}=12 \mathrm{mV}, V_{4}=17.4 \mathrm{mV}, \tau_{\max }=14.925 \mathrm{~ms}$. These parameters result in the resting potential value $V_{\text {rest }}=-59.47 \mathrm{mV}$, which is the solution of equation $I_{i o n}\left(V, w_{\infty}(V)\right)=0$ and is very close to $V_{L}$ value. The initial conditions for all numerical simulations of the ML model in this paper are as follows: $V(t=0)=V_{\text {rest }}, w(t=0)=w_{\infty}\left(V_{\text {rest }}\right)$.

\section{Linearization of the Morris-Lecar equations at large constant stimulation}

In what follows, we consider the case $I_{\text {stim }}>I_{\max }$ and seek a solution for the potential in the form $V(t)=V_{s t}+U(t)$, where $V_{s t}$ is the stationary potential value determined from equation $I_{i o n}\left(V_{s t}, w_{\infty}\left(V_{s t}\right)\right)=I_{\text {stim }}$ and $U(t \rightarrow+\infty)=0$. In addition, we assume that for any moment of time $t$ the condition $|U(t)| \ll\left|V_{s t}\right|$ holds. Given this, we expand $w_{\infty}(V)$ and $m_{\infty}(V)$ into a Taylor series up to the linear term with respect to $U$ :

$$
\begin{aligned}
& w_{\infty}\left(V_{s t}+U\right) \approx w_{\infty}\left(V_{s t}\right)+\frac{d w_{\infty}\left(V_{s t}\right)}{d V} U=a+b \cdot U, \\
& m_{\infty}\left(V_{s t}+U\right) \approx m_{\infty}\left(V_{s t}\right)+\frac{d m_{\infty}\left(V_{s t}\right)}{d V} U=p+q \cdot U,
\end{aligned}
$$

where $a=w_{\infty}\left(V_{s t}\right), b=d w_{\infty}\left(V_{s t}\right) / d V, p=m_{\infty}\left(V_{s t}\right), q=d m_{\infty}\left(V_{s t}\right) / d V$.

Next, we exactly solve the linearized ML equation on $w$, assuming that $\tau(V)=$ const $=\tau\left(V_{s t}\right) \equiv \tau$,

$$
\left\{\begin{array}{l}
\tau d w / d t=a+b U(t)-w \\
w\left(t=t_{0}\right)=w_{0}
\end{array}\right.
$$


Its general solution has form

$$
w(t)=a+W_{0}(t)+b \exp \left(-\frac{t}{\tau}\right) \int_{t_{0}}^{t} \exp \left(\frac{t^{\prime}}{\tau}\right) \frac{U\left(t^{\prime}\right)}{\tau} d t^{\prime}
$$

where $W_{0}(t)=\left(w_{0}-a\right) \exp \left(-\left(t-t_{0}\right) / \tau\right)$.

We find value $w\left(t_{0}\right)=w_{0}$ in a local extremum point of the potential $V\left(t=t_{0}\right)=V_{0}=V_{s t}+U_{0}$, which is determined by condition $\frac{d V}{d t}\left(t=t_{0}\right)=0$. One gets

$$
w_{0}=\frac{I_{s t i m}-g_{C a} \cdot m_{\infty}\left(V_{0}\right) \cdot\left(V_{0}-V_{C a}\right)-g_{L} \cdot\left(V_{0}-V_{L}\right)}{g_{K} \cdot\left(V_{0}-V_{K}\right)} .
$$

Further, writing explicitly the equation on $U$ and neglecting nonlinear terms, we obtain a linear integro-differential equation for the potential $U$

$$
\frac{d U}{d t}=-G(t)-A(t) U-B \exp \left(-\frac{t}{\tau}\right) \int_{t_{0}}^{t} \exp \left(\frac{t^{\prime}}{\tau}\right) \frac{U\left(t^{\prime}\right)}{\tau} d t^{\prime}
$$

where coefficients $A(t), B$, and $G(t)$ are as follows:

$$
\begin{aligned}
A(t) & =\left[g_{C a}\left(p+q\left(V_{s t}-V_{C a}\right)\right)+g_{K}\left(a+W_{0}(t)\right)+g_{L}\right] / C_{m} \equiv A+A_{0}(t), \\
A & =\left[g_{C a}\left(p+q\left(V_{s t}-V_{C a}\right)\right)+g_{K} a+g_{L}\right] / C_{m} \\
A_{0}(t) & =g_{K} W_{0}(t) / C_{m} \equiv A_{K} \exp \left(-\left(t-t_{0}\right) / \tau\right), \quad A_{K}=g_{K}\left(w_{0}-a\right) / C_{m} \\
B & =g_{K} b\left(V_{s t}-V_{K}\right) / C_{m} \\
G(t) & =A_{0}(t)\left(V_{s t}-V_{K}\right)=B\left(\left(w_{0}-a\right) / b\right) \exp \left(-\left(t-t_{0}\right) / \tau\right)
\end{aligned}
$$

Integrating by parts, we obtain

$$
\frac{d U}{d t}=G_{1}(t)-A_{1}(t) U(t)+B \exp \left(-\frac{t}{\tau}\right) \int_{t_{0}}^{t} \exp \left(\frac{t^{\prime}}{\tau}\right) \frac{d U}{d t^{\prime}} d t^{\prime}
$$

where $G_{1}(t)=-G(t)+B U\left(t_{0}\right) \exp \left(-\left(t-t_{0}\right) / \tau\right)$ and $A_{1}(t)=A(t)+B$.

Further, given that $U(t)=U\left(t_{0}\right)+\int_{t_{0}}^{t}\left(d U / d t^{\prime}\right) d t^{\prime}$, one can reduce the previous equation on $U$ to an integral equation for its derivative $f(t)=d U / d t$,

$$
f(t)=G_{2}(t)+\int_{t_{0}}^{t} K\left(t, t^{\prime}\right) f\left(t^{\prime}\right) d t^{\prime},
$$

where $G_{2}(t)=G_{1}(t)-A_{1}(t) U\left(t_{0}\right)=G_{3}+G_{4} \exp \left(-\left(t-t_{0}\right) / \tau\right), G_{3}=-(A+B) U\left(t_{0}\right), G_{4}=U\left(t_{0}\right)\left(-A_{K}+B\right)-B\left(w_{0}-\right.$ $a) / b$, and

$$
K\left(t, t^{\prime}\right)=-A_{1}(t)+B \exp \left(-\frac{\left(t-t^{\prime}\right)}{\tau}\right)=-(A+B)-A_{K} \exp \left(-\frac{\left(t-t_{0}\right)}{\tau}\right)+B \exp \left(-\frac{\left(t-t^{\prime}\right)}{\tau}\right) .
$$

The resulting equation (11) for $f(t)$ is an inhomogeneous Volterra integral equation of the second kind. Twice differentiating both sides of Eq. (11) with respect to $t$, we obtain that the integral equation (11) is equivalent to ordinary differential equation of the second order

$$
\frac{d^{2} f}{d t^{2}}+\left(2 \gamma+A_{K} \exp \left(-\left(t-t_{0}\right) / \tau\right)\right) \frac{d f}{d t}+\left(-\frac{A_{K}}{\tau} \exp \left(-\left(t-t_{0}\right) / \tau\right)+\omega_{0}^{2}\right) f(t)=0
$$

where constants $2 \gamma=A+1 / \tau$ and $\omega_{0}^{2}=(A+B) / \tau$ have been introduced. 

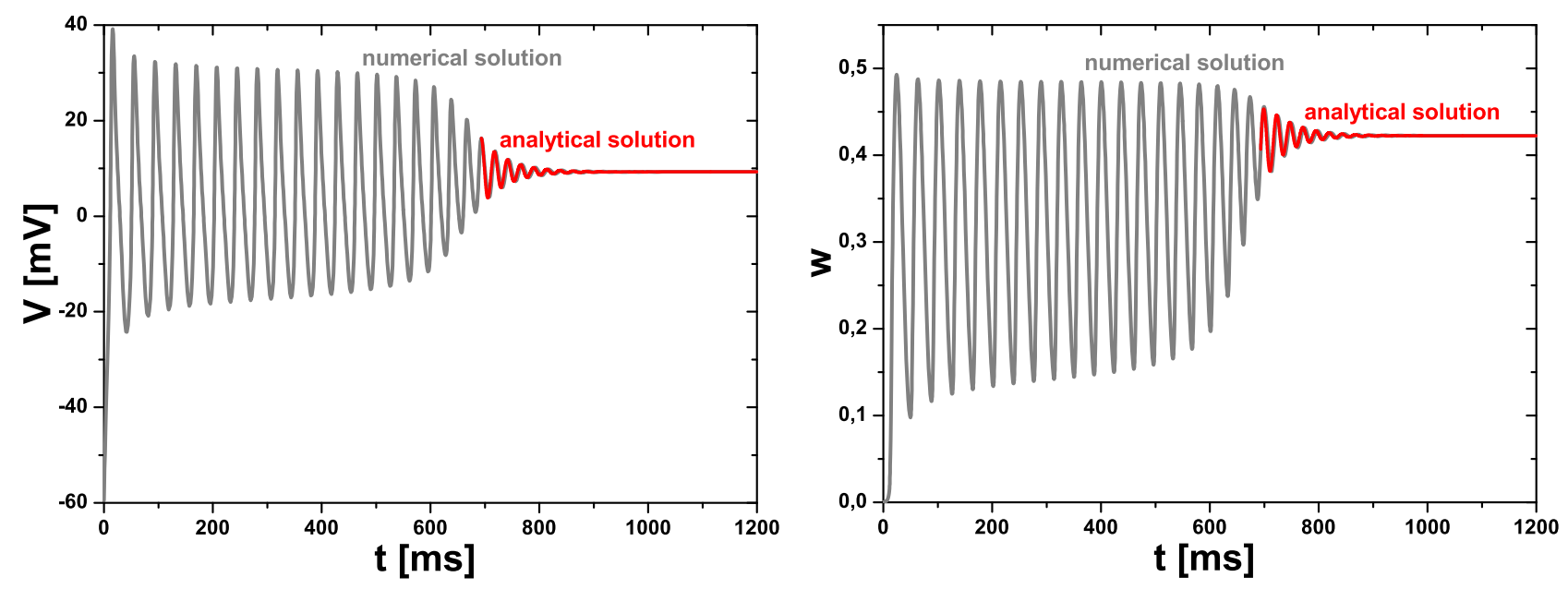

Figure 2. Left graph: The gray curve is a numerical solution for dynamics of neuron potential $V(t)$ in the Morris-Lecar model with the 1st excitability type at $I_{\text {stim }}=116.3 \mu \mathrm{A} / \mathrm{cm}^{2}>I_{\max }=116.1 \mu \mathrm{A} / \mathrm{cm}^{2}$. The red curve is an analytical solution of the linearized system of equations of the Morris-Lecar model with initial conditions taken at the point of a local maximum of the potential $\left(t_{0}=693.3 \mathrm{~ms}, V_{0}=16.35 \mathrm{mV}\right)$. Right graph: The corresponding numerical (gray) and analytical (red) solutions for $w(t)$. Parameters of the analytical formulas for this example are as follows: $V_{s t}=9.28 \mathrm{mV}, a=0.42, \omega_{0}=262.1 \mathrm{~Hz}$, $\gamma=21.3 \mathrm{~Hz}, \omega=261.2 \mathrm{~Hz}, 1 / \tau=67.2 \mathrm{~Hz}, \eta=0.08, \chi=0.17,2 \gamma /\left|A_{K}\right|=6.78, U_{0}=7.07 \mathrm{mV}, a / w_{0}=1.04, W_{a}=0.05$, and $W_{c}=-0.02$, where the last five parameters depend on $t_{0}$ and $V_{0}$ values.

Returning to potential $U$ and allocating the full derivative, we have

$$
\frac{d^{2} U}{d t^{2}}+\left(2 \gamma+A_{K} \exp \left(-\left(t-t_{0}\right) / \tau\right)\right) \frac{d U}{d t}+\omega_{0}^{2} U=\text { const }
$$

Assuming that potential $U(t)$ and all its derivatives tend to zero at $t \rightarrow+\infty$, one gets const $=0$. Finally, we obtain

$$
\frac{d^{2} U}{d t^{2}}+\left(2 \gamma+A_{K} \exp \left(-\left(t-t_{0}\right) / \tau\right)\right) \frac{d U}{d t}+\omega_{0}^{2} U=0
$$

with initial conditions

$$
U\left(t=t_{0}\right)=U_{0}=V_{0}-V_{s t}, \quad \frac{d U}{d t}\left(t=t_{0}\right)=0 .
$$

\section{Analytical solution of the linearized equations}

Assuming that $w_{0} \approx a$ and neglecting the time-dependent parameter in Eq. (15), we arrive at

$$
\frac{d^{2} U}{d t^{2}}+2 \gamma \frac{d U}{d t}+\omega_{0}^{2} U(t)=0
$$

Given the initial conditions (16), the solution of Eq. (17) has form

$$
U(t)=U_{0} \exp \left(-\gamma\left(t-t_{0}\right)\right)\left[\cos \left(\omega\left(t-t_{0}\right)\right)+\frac{\gamma}{\omega} \sin \left(\omega\left(t-t_{0}\right)\right)\right]
$$

with angular frequency $\omega=\sqrt{\omega_{0}^{2}-\gamma^{2}}$ and oscillation period $T=2 \pi / \omega$. This solution describes exponentiallydamped harmonic oscillations and corresponds well with the numerical result (left graph in Fig. 2 and two left graphs in Fig. 3). It is worth noting that $\omega_{0}$ and $\gamma$ are independent of $t_{0}$ and $V_{0}$. Therefore the dependencies $\omega_{0}\left(I_{\text {stim }}\right)$, $\gamma\left(I_{\text {stim }}\right)$, and $\omega\left(I_{\text {stim }}\right)$ are relatively universal and, moreover, these can be continued in the range $I_{\text {stim }}<I_{\max }$ (Fig. 3 , right graph), though in this case there is no correspondence between the numerical and analytical solutions.

One can also obtain an explicit solution for $w(t)$ by substituting $U(t)$ into Eq. (7):

$$
w(t)=a+\left(w_{0}-a+w_{1}\right) \exp \left(-\left(t-t_{0}\right) / \tau\right)+w_{1} \exp \left(-\gamma\left(t-t_{0}\right)\right)\left[-\cos \left(\omega\left(t-t_{0}\right)\right)+H \sin \left(\omega\left(t-t_{0}\right)\right)\right],
$$



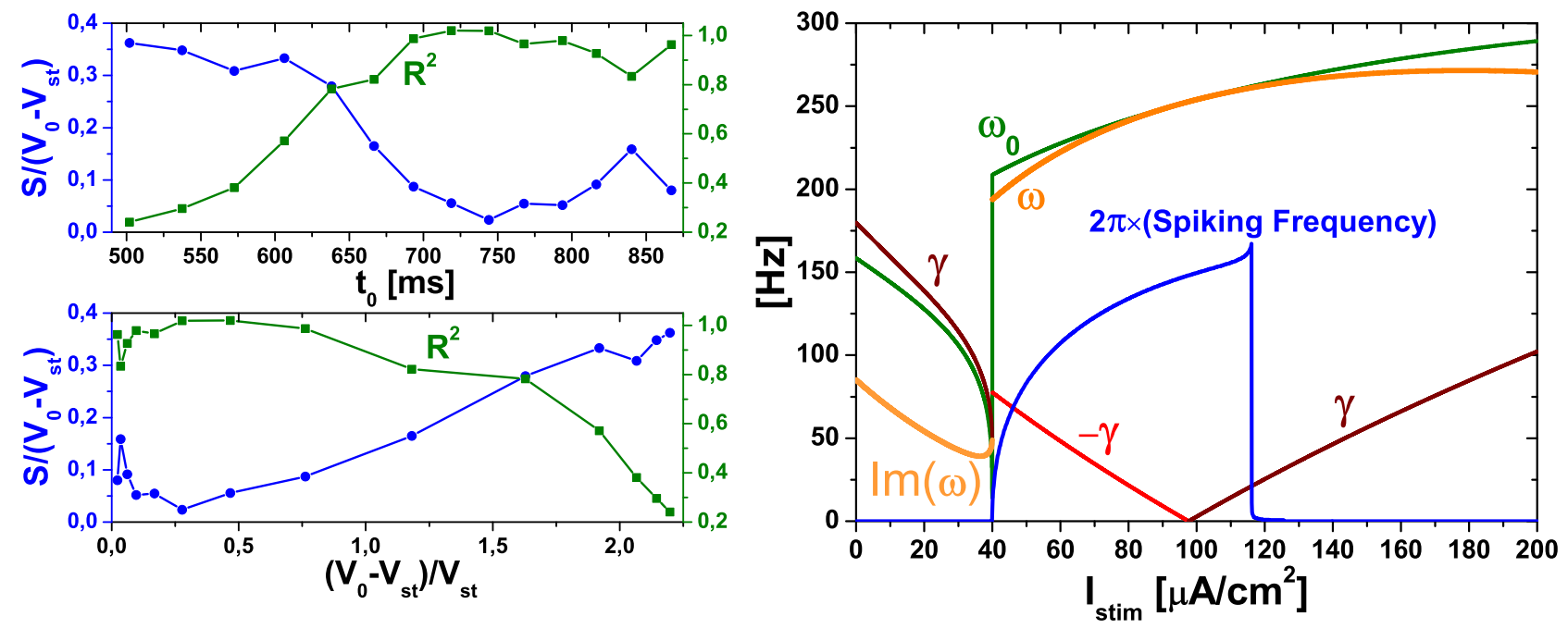

Figure 3. Left graph: Quantitative evaluation of the correspondence between the numerical and analytical solutions at $I_{\text {stim }}$ $=116.3 \mu \mathrm{A} / \mathrm{cm}^{2}$ (see left graph in Fig. 2): dependencies for $S=\frac{1}{n} \sum_{i=1}^{n}\left|V_{s t}+U\left(t_{i}\right)-V\left(t_{i}\right)\right|$ (blue circles, left scale) and $R^{2}=\frac{\sum_{i=1}^{n}\left(V_{s t}+U\left(t_{i}\right)-V_{\text {mean }}\right)^{2}}{\sum_{i=1}^{n}\left(V\left(t_{i}\right)-V_{\text {mean }}\right)^{2}}$ (green squares, right scale) on different values of $t_{0}$ and $V_{0}$. Here $V_{\text {mean }}=\frac{1}{n} \sum_{i=1}^{n} V\left(t_{i}\right)$ and $\left\{t_{i}\right\}_{i=1}^{n}$ is the set of time moments $t_{i}>t_{0}$, for which numerical solution $V\left(t_{i}\right)$ is known. As one can see from the lower graph, an approximate empirical condition of the good correspondence is $V_{0}<2 V_{s t}$. Right graph: Analytical dependencies of $\omega_{0}$, $\gamma$, and $\omega$ on the value of constant stimulating current $I_{\text {stim }}$, with superimposed spiking frequency from Fig. 1.

where $w_{1}=b U_{0} A / B$ and $H=\omega_{0}^{2} /(\omega A)-\gamma / \omega$.

Using auxiliary trigonometric transformations, one can write functions $U(t)$ and $w(t)$ in a more compact form. Denoting

$$
\cos (\eta)=\frac{1}{\sqrt{1+(\gamma / \omega)^{2}}}=\omega / \omega_{0}, \quad \sin (\eta)=\frac{\gamma / \omega}{\sqrt{1+(\gamma / \omega)^{2}}}=\gamma / \omega_{0}
$$

we get

$$
U(t)=U_{0} \exp \left(-\gamma\left(t-t_{0}\right)\right) \frac{\cos \left(\omega\left(t-t_{0}\right)-\eta\right)}{\cos (\eta)}
$$

where $\eta=\arctan (\gamma / \omega)$ is the inverse function of $\tan (\eta)=\gamma / \omega$. This expression for $U(t)$ is completely equivalent to the previous solution (18).

In turn, introducing notations

$$
s=(1-\gamma \tau) /(\omega \tau), \quad \cos (\chi)=1 / \sqrt{1+s^{2}}, \quad \sin (\chi)=s / \sqrt{1+s^{2}},
$$

we obtain a compact solution for $w(t)$,

$$
w(t)=a+W_{c} \exp \left(-\left(t-t_{0}\right) / \tau\right)+W_{a} \exp \left(-\gamma\left(t-t_{0}\right)\right) \sin \left(\omega\left(t-t_{0}\right)+\chi-\eta\right)
$$

where $\chi=\arctan (s)$, quantity $W_{a}=\left(b U_{0} /(\omega \tau)\right) \sqrt{1+A / B}$ determines the amplitude of the damped oscillations of $w(t)$ (see Fig. 2, right graph), and $W_{c}=\left(w_{0}-a\right)-W_{a} \sin (\chi-\eta)$.

\section{Conclusion}

We have shown analytically, and confirmed numerically, that for the Morris-Lecar neuron model upon stimulation by large constant depolarizing current the later stages of spike amplitude damping can be accurately reduced to exponentially damped harmonic oscillations. The results may be useful as a complementary to bifurcation analysis, in particular, for shaping the applicability boundary of linear perturbation method, which implies exponential damping, in studies of dynamical state stability. 


\section{Acknowledgments}

This work was partially funded by the Russian Foundation for Basic Research according to the research project \# 17-29-07093.

\section{References}

[1] C. Morris, H. Lecar, Voltage oscillations in the barnacle giant muscle fiber, Biophys. J. 35, 193-213 (1981). https://doi.org/10.1016/S0006-3495(81)84782-0

[2] J. Rinzel, B. Ermentrout, Analysis of neural excitability and oscillations, In: C. Koch, I. Segev (Eds.), Methods in neuronal modeling: From synapses to networks (2nd Ed., MIT Press, 1998), pp. 251-291. https://www.researchgate.net/publication/237128320

[3] T. Tateno, A. Harsch, H. Robinson, Threshold firing frequency-current relationships of neurons in rat somatosensory cortex: type 1 and type 2 dynamics, J. Neurophysiol. 92, 2283-2294 (2004). https://doi.org/10.1152/jn.00109.2004

[4] K. Tsumoto et al., Bifurcations in Morris-Lecar neuron model, Neurocomputing 69, 293-316 (2006). https://doi.org/10.1016/j.neucom.2005.03.006

[5] L.H. Nguyen, K.S. Hong, S. Park, Bifurcation control of the Morris-Lecar neuron model via a dynamic state-feedback control, Biol. Cybern. 106, 587-594 (2012). https://doi.org/10.1007/s00422-012-0508-4

[6] T. Zemskova, A. Paraskevov. On damping mechanism of periodic spiking in Morris-Lecar model at large values of constant stimulating current. Bernstein Conference 2018. https://doi.org/10.6084/m9.figshare.11301776

[7] C. Koch, Biophysics of Computation: Information Processing in Single Neurons (Oxford University Press, 1999). https://global .oup.com/academic/product/biophysics-of-computation-9780195181999

[8] E.M. Izhikevich, Dynamical Systems in Neuroscience: The Geometry of Excitability and Bursting (1st Ed., MIT Press, 2007).

https://mitpress.mit.edu/books/dynamical-systems-neuroscience

[9] D. Sterratt, B. Graham, A. Gillies, D. Willshaw, Principles of Computational Modelling in Neuroscience (Cambridge University Press, 2011). https://doi.org/10.1017/CB09780511975899

[10] G.B. Ermentrout, D.H. Terman, Mathematical Foundations of Neuroscience (Springer-Verlag New York, 2010). https://doi.org/10.1007/978-0-387-87708-2

[11] W. Gerstner, W.M. Kistler, R. Naud, L. Paninski, Neuronal Dynamics: From Single Neurons to Networks and Models of Cognition (Cambridge University Press, 2014). https://doi.org/10.1017/СB09781107447615

[12] J.M. Gonzalez-Miranda, Pacemaker dynamics in the full Morris-Lecar model, Commun. Nonlinear Sci. Numer. Simulat. 19, 3229-3241 (2014). https://doi.org/10.1016/j.cnsns. 2014.02.020 